\title{
On Generalized Fractional Kinetic Equations Involving Generalized Bessel Function of the First Kind
}

\author{
Dinesh Kumar, ${ }^{1}$ S. D. Purohit, ${ }^{2}$ A. Secer, ${ }^{3}$ and A. Atangana ${ }^{4}$ \\ ${ }^{1}$ Department of Mathematics \& Statistics, J. N. Vyas University, Jodhpur 342001, India \\ ${ }^{2}$ Department of Basic Sciences (Mathematics), College of Technology and Engineering, M. P. University of Agriculture and Technology, \\ Udaipur, Rajasthan 313001, India \\ ${ }^{3}$ Department of Mathematical Engineering, Yildiz Technical University, Davutpasa, 34210 Istanbul, Turkey \\ ${ }^{4}$ Institute for Groundwater Studies, University of the Free State, Bloemfontein 9300, South Africa
}

Correspondence should be addressed to A. Atangana; abdonatangana@yahoo.fr

Received 18 July 2014; Revised 1 September 2014; Accepted 2 September 2014

Academic Editor: Hossein Jafari

Copyright (C) 2015 Dinesh Kumar et al. This is an open access article distributed under the Creative Commons Attribution License, which permits unrestricted use, distribution, and reproduction in any medium, provided the original work is properly cited.

We develop a new and further generalized form of the fractional kinetic equation involving generalized Bessel function of the first kind. The manifold generality of the generalized Bessel function of the first kind is discussed in terms of the solution of the fractional kinetic equation in the paper. The results obtained here are quite general in nature and capable of yielding a very large number of known and (presumably) new results.

\section{Introduction and Preliminaries}

Bessel functions are playing the important role in studying solutions of differential equations, and they are associated with a wide range of problems in important areas of mathematical physics, like problems of acoustics, radiophysics, hydrodynamics, and atomic and nuclear physics. These considerations have led various workers in the field of special functions to explore the possible extensions and applications of the Bessel functions. Among many properties of Bessel functions, they also have investigated some possible extensions of the Bessel functions.

The generalized Bessel function of the first kind $\omega_{p}(z)$ is defined for $z \in \mathbb{C} \backslash\{0\}$ and $b, c, p \in \mathbb{C}(\Re(p)>-1)$ by the following series [1, page 10, (1.15)] (for recent work, see also [2-6]):

$$
\omega_{p, b, c}(z)=\omega_{p}(z)=\sum_{k=0}^{\infty} \frac{(-1)^{k} c^{k}}{k ! \Gamma(p+(b+1) / 2+k)}\left(\frac{z}{2}\right)^{2 k+p}
$$

where $\mathbb{C}$ denotes the set of complex numbers and $\Gamma(a)$ is the familiar Gamma function.
The special cases of series (1) can be obtained as follows.

(i) If we put $b=c=1$ in (1), then we obtain the familiar Bessel function of the first kind [7] of order $p$ for $z, p \in \mathbb{C}$ with $\mathfrak{R}(p)>-1$ defined and represented by the following expressions (see also $[1,8]$ ):

$$
J_{p}(z)=\sum_{k=0}^{\infty} \frac{(-1)^{k}}{k ! \Gamma(p+k+1)}\left(\frac{z}{2}\right)^{2 k+p}, \quad z \in \mathbb{C} .
$$

(ii) Putting $b=1$ and $c=-1$ in series (1), we get the modified Bessel function of the first kind of order $p$ defined by (see $[1,7])$

$$
I_{p}(z)=\sum_{k=0}^{\infty} \frac{1}{k ! \Gamma(p+k+1)}\left(\frac{z}{2}\right)^{2 k+p}, \quad z \in \mathbb{C} ;
$$

the series given by (3) is also a special case of Galués generalized modified Bessel function [9] depending on parameters $a=0,1,2, \ldots$ and $p>-1$, given as follows:

$$
{ }_{a} I_{p}(z)=\sum_{k=0}^{\infty} \frac{(z / 2)^{2 k+p}}{k ! \Gamma(p+a k+1)}, \quad z \in \mathbb{C} .
$$


(iii) Letting $b=2$ and $c=1$ in series (1), we have the spherical Bessel function of the first kind of order $p$ defined by (see [1])

$$
j_{p}(z)=\sqrt{\frac{\pi}{2}} \sum_{k=0}^{\infty} \frac{(-1)^{k}}{k ! \Gamma(p+k+3 / 2)}\left(\frac{z}{2}\right)^{2 k+p}, \quad z \in \mathbb{C} .
$$

Furthermore, Deniz et al. [10] considered the function $\varphi_{p, b, c}(z)$, defined in terms of the generalized Bessel function $\omega_{p}(z)$, by the transformation

$$
\begin{aligned}
\varphi_{p, b, c}(z) & =2^{p} \Gamma\left(p+\frac{b+1}{2}\right) z^{1-p / 2} \omega_{p}(\sqrt{z}) \\
& =z+\sum_{k=1}^{\infty} \frac{(-c)^{k}}{4^{k}(\nu)_{k}} \frac{z^{k+1}}{k !},
\end{aligned}
$$

where $v=p+(b+1) / 2 \notin Z_{0}^{-}:=\{0,-1,-2, \ldots\}$ and $(a)_{k}$ is the Pochhammer symbol defined (for $a \in \mathbb{C}$ ) by

$$
\begin{aligned}
(a)_{k} & := \begin{cases}1 & (k=0) \\
a(a+1) \cdots(a+k-1) & (k \in N:=\{1,2,3, \ldots\})\end{cases} \\
& =\frac{\Gamma(a+k)}{\Gamma(a)} \quad\left(a \in \mathbb{C} \backslash Z_{0}^{-}\right) .
\end{aligned}
$$

Fractional differential equations appear more and more frequently for modeling of relevant systems in several fields of applied sciences. These equations play important roles, not only in mathematics, but also in physics, dynamical systems, control systems, and engineering, to create the mathematical model of many physical phenomena. In particular, the kinetic equations describe the continuity of motion of substance and are the basic equations of mathematical physics and natural science. Therefore, in literature we found several papers that analyze extensions and generalizations of these equations involving various fractional calculus operators. One may, for instance, refer to such type of works by [11-23].

Haubold and Mathai [13] have established a functional differential equation between rate of change of reaction, the destruction rate, and the production rate as follows:

$$
\frac{d N}{d t}=-d\left(N_{t}\right)+p\left(N_{t}\right)
$$

where $N=N(t)$ is the rate of reaction, $d=d(N)$ is the rate of destruction, $p=p(N)$ is the rate of production, and $N_{t}$ denotes the function defined by $N_{t}\left(t^{*}\right)=N\left(t-t^{*}\right), t^{*}>0$.

Haubold and Mathai studied a special case of (8), when spatial fluctuations or inhomogeneities in the quantity $N(t)$ are neglected, is given by the equation

$$
\frac{d N_{i}}{d t}=-c_{i} N_{i}(t)
$$

together with the initial condition that $N_{i}(t=0)=N_{0}$, is the number of density of species $i$ at time $t=0, c_{i}>0$. If we decline the index $i$ and integrate the standard kinetic equation (9), we have

$$
N(t)-N_{0}=-c_{0} D_{t}^{-1} N(t)
$$

where ${ }_{0} D_{t}^{-1}$ is the special case of the Riemann-Liouville integral operator ${ }_{0} D_{t}^{-v}$ defined as

$$
{ }_{0} D_{t}^{-\nu} f(t)=\frac{1}{\Gamma(\nu)} \int_{0}^{t}(t-s)^{\nu-1} f(s) d s, \quad t>0, \mathfrak{R}(\nu)>0 .
$$

Haubold and Mathai [13] have given the fractional generalization of the standard kinetic equation (10) as

$$
N(t)-N_{0}=-c^{\nu}{ }_{0} D_{t}^{-v} N(t)
$$

and have provided the solution of (12) as follows:

$$
N(t)=N_{0} \sum_{k=0}^{\infty} \frac{(-1)^{k}}{\Gamma(\nu k+1)}(c t)^{\nu k}
$$

Further, Saxena and Kalla [17] considered the following fractional kinetic equation:

$$
N(t)-N_{0} f(t)=-c^{\nu}\left({ }_{0} D_{t}^{-\nu} N\right)(t), \quad(\Re(\nu)>0),
$$

where $N(t)$ denotes the number density of a given species at time $t, N_{0}=N(0)$ is the number density of that species at time $t=0, c$ is a constant, and $f \in L(0, \infty)$.

By applying the Laplace transform to (14), we have

$$
\begin{aligned}
L & {[N(t)](p) } \\
& =N_{0} \frac{F(p)}{1+c^{v} p^{-v}} \\
& =N_{0}\left(\sum_{n=0}^{\infty}\left(-c^{v}\right)^{n} p^{-n v}\right) F(p) \quad\left(n \in N_{0},\left|\frac{c}{p}\right|<1\right),
\end{aligned}
$$

where the Laplace transform [24] is defined by

$$
F(p)=L[f(t)]=\int_{0}^{\infty} e^{-p t} f(t) d t, \quad \Re(p)>0 .
$$

The aim of this paper is to develop a new and further generalized form of the fractional kinetic equation involving generalized Bessel function of the first kind. The manifold generality of the generalized Bessel function of the first kind is discussed in terms of the solution of the above fractional kinetic equation. Moreover, the results obtained here are quite capable of yielding a very large number of known and (presumably) new results.

\section{Solution of Generalized Fractional Kinetic Equations}

In this section, we will investigate the solution of the generalized fractional kinetic equations. The results are as follows. 
Theorem 1. If $d>0, v>0, c, b, l, t \in \mathbb{C}$, and $\mathfrak{R}(l)>-1$, then for the solution of the equation

$$
N(t)-N_{0} \omega_{l, b, c}(t)=-d^{v}{ }_{0} D_{t}^{-v} N(t),
$$

there holds the formula:

$$
\begin{aligned}
N(t)= & N_{0} \sum_{k=0}^{\infty} \frac{(-c)^{k} \Gamma(2 k+l+1)}{k ! \Gamma(l+k+(b+1) / 2)}\left(\frac{t}{2}\right)^{2 k+l} \\
& \times E_{\gamma, 2 k+l+1}\left(-d^{\nu} t^{\nu}\right),
\end{aligned}
$$

where $E_{\gamma, 2 k+l+1}(\cdot)$ is the generalized Mittag-Leffler function [25].

Proof. The Laplace transform of the Riemann-Liouville fractional integral operator is given by $[26,27]$

$$
L\left\{{ }_{0} D_{t}^{-v} f(t) ; p\right\}=p^{-v} F(p)
$$

where $F(p)$ is defined in (16). Now, applying the Laplace transform to both sides of (17), we get

$$
\begin{aligned}
L & {[N(t) ; p] } \\
& =N_{0} L\left[\omega_{l, b, c}(t) ; p\right]-d^{v} L\left[{ }_{0} D_{t}^{-v} N(t) ; p\right],
\end{aligned}
$$$$
N(p)
$$

$$
\begin{aligned}
=N_{0} \int_{0}^{\infty} & e^{-p t} \sum_{k=0}^{\infty} \frac{(-c)^{k}}{k ! \Gamma(l+k+(b+1) / 2)}\left(\frac{t}{2}\right)^{2 k+l} \\
& -d^{v} p^{-v} N(p),
\end{aligned}
$$

$$
\begin{aligned}
& N(p)\left[1+d^{v} p^{-v}\right] \\
& =N_{0} \sum_{k=0}^{\infty} \frac{(-c)^{k} 2^{-(2 k+l)}}{k ! \Gamma(l+k+(b+1) / 2)} \int_{0}^{\infty} e^{-p t} t^{2 k+l} d t \\
& =N_{0} \sum_{k=0}^{\infty} \frac{(-c)^{k} 2^{-(2 k+l)}}{k ! \Gamma(l+k+(b+1) / 2)} \frac{\Gamma(2 k+l+1)}{p^{2 k+l+1}}
\end{aligned}
$$

$N(p)$

$$
\begin{aligned}
= & N_{0} \sum_{k=0}^{\infty} \frac{(-c)^{k} 2^{-(2 k+l)} \Gamma(2 k+l+1)}{k ! \Gamma(l+k+(b+1) / 2)} \\
& \times\left\{p^{-(2 k+l+1)} \sum_{r=0}^{\infty} \frac{(1)_{r}\left[-(p / d)^{-v}\right]^{r}}{(r) !}\right\} .
\end{aligned}
$$

Taking Laplace inverse of (20) and using $L^{-1}\left\{p^{-\nu}\right\}=t^{\nu-1} / \Gamma(\nu)$, $\mathfrak{R}(\nu)>0$, we have

$$
\begin{aligned}
L^{-1}\{N(p)\} \\
=N_{0} \sum_{k=0}^{\infty} \frac{(-c)^{k} 2^{-(2 k+l)} \Gamma(2 k+l+1)}{k ! \Gamma(l+k+(b+1) / 2)} \\
\quad \times L^{-1}\left\{\sum_{r=0}^{\infty}(-1)^{r} d^{v r} p^{-(2 k+l+v r+1)}\right\},
\end{aligned}
$$

$N(t)$

$$
\begin{aligned}
= & N_{0} \sum_{k=0}^{\infty} \frac{(-c)^{k} 2^{-(2 k+l)} \Gamma(2 k+l+1)}{k ! \Gamma(l+k+(b+1) / 2)} \\
& \times\left\{\sum_{r=0}^{\infty}(-1)^{r} d^{\nu r} \frac{t^{(2 k+l+v r)}}{\Gamma(\nu r+2 k+l+1)}\right\} \\
= & N_{0} \sum_{k=0}^{\infty} \frac{(-c)^{k} 2^{-(2 k+l)} \Gamma(2 k+l+1)}{k ! \Gamma(l+k+(b+1) / 2)} t^{2 k+l} \\
& \times\left\{\sum_{r=0}^{\infty}(-1)^{r} d^{\nu r} \frac{t^{\nu r}}{\Gamma(\nu r+2 k+l+1)}\right\} \\
= & N_{0} \sum_{k=0}^{\infty} \frac{(-c)^{k} \Gamma(2 k+l+1)}{k ! \Gamma(l+k+(b+1) / 2)}\left(\frac{t}{2}\right)^{2 k+l} \\
& \times\left\{\sum_{r=0}^{\infty}(-1)^{r} d^{\nu r} \frac{t^{\nu r}}{\Gamma(\nu r+2 k+l+1)}\right\},
\end{aligned}
$$

$N(t)$

$$
\begin{aligned}
= & N_{0} \sum_{k=0}^{\infty} \frac{(-c)^{k} \Gamma(2 k+l+1)}{k ! \Gamma(l+k+(b+1) / 2)}\left(\frac{t}{2}\right)^{2 k+l} \\
& \times E_{v, 2 k+l+1}\left(-d^{\nu} t^{\nu}\right) .
\end{aligned}
$$

This completes the proof of Theorem 1 .

If we set $b=c=1$ in (17), then generalized Bessel function $\omega_{l, b, c}(z)$ reduces to Bessel function of the first kind $J_{l}(z)$ given by (2), and we arrive at the following result.

Corollary 2. If $d>0, v>0, l, t \in \mathbb{C}$, and $\mathfrak{R}(l)>-1$, then for the solution of the equation

$$
N(t)-N_{0} J_{l}(t)=-d^{v}{ }_{0} D_{t}^{-v} N(t),
$$

there holds the formula:

$$
\begin{aligned}
N(t)= & N_{0} \sum_{k=0}^{\infty} \frac{(-1)^{k} \Gamma(2 k+l+1)}{k ! \Gamma(l+k+1)}\left(\frac{t}{2}\right)^{2 k+l} \\
& \times E_{v, 2 k+l+1}\left(-d^{\nu} t^{\nu}\right) .
\end{aligned}
$$


Further, taking $b=1$ and $c=-1$ in (17), then we obtain result of generalized fractional kinetic equation having modified Bessel function of the first kind.

Corollary 3. If $d>0, v>0, l, t \in \mathbb{C}$, and $\mathfrak{R}(l)>-1$, then for the solution of the equation

$$
N(t)-N_{0} I_{l}(t)=-d^{v}{ }_{0} D_{t}^{-v} N(t),
$$

there holds the formula:

$$
\begin{aligned}
N(t)= & N_{0} \sum_{k=0}^{\infty} \frac{\Gamma(2 k+l+1)}{k ! \Gamma(l+k+1)}\left(\frac{t}{2}\right)^{2 k+l} \\
& \times E_{v, 2 k+l+1}\left(-d^{v} t^{\nu}\right) .
\end{aligned}
$$

Letting $b=2$ and $c=1$ in (17), then generalized Bessel function $\omega_{l, b, c}(z)$ reduces to the spherical Bessel function of the first kind $j_{l}(z)$ given by (5), and we obtain the following interesting result.

Corollary 4. If $d>0, v>0, l, t \in \mathbb{C}$, and $\mathfrak{R}(l)>-1$, then for the solution of the equation

$$
N(t)-N_{0} j_{l}(t)=-d^{v}{ }_{0} D_{t}^{-v} N(t),
$$

there holds the solution of (22)

$$
\begin{aligned}
N(t)= & N_{0} \sqrt{\frac{\pi}{2}} \sum_{k=0}^{\infty} \frac{(-1)^{k} \Gamma(2 k+l+1)}{k ! \Gamma(l+k+3 / 2)}\left(\frac{t}{2}\right)^{2 k+l} \\
& \times E_{v, 2 k+l+1}\left(-d^{\nu} t^{\nu}\right) .
\end{aligned}
$$

Theorem 5. If $d>0, v>0, c, b, l, t \in \mathbb{C}$, and $\Re(l)>-1$, then for the solution of the equation

$$
N(t)-N_{0} \omega_{l, b, c}\left(d^{v} t^{\nu}\right)=-d^{v}{ }_{0} D_{t}^{-v} N(t),
$$

there holds the formula:

$$
\begin{aligned}
N(t)= & N_{0} \sum_{k=0}^{\infty} \frac{(-c)^{k} \Gamma(2 k v+v l+1)}{k ! \Gamma(l+k+(b+1) / 2)}\left(\frac{d^{v} t^{v}}{2}\right)^{2 k+l} \\
& \times E_{\gamma,(2 k+l) v+1}\left(-d^{v} t^{\nu}\right),
\end{aligned}
$$

where $E_{\gamma, 2 k v+\nu l+1}(\cdot)$ is the generalized Mittag-Leffler function.

Proof. The Laplace transform of the Riemann-Liouville fractional integral operator is given by [26]

$$
L\left\{{ }_{0} D_{t}^{-v} f(t) ; p\right\}=p^{-v} F(p),
$$

where $F(p)$ is defined in (16). Now, applying the Laplace transform to both sides of (28), we get

$$
\begin{aligned}
& L[N(t) ; p] \\
& =N_{0} L\left[\omega_{l, b, c}\left(d^{v} t^{\nu}\right) ; p\right]-d^{v} L\left[{ }_{0} D_{t}^{-v} N(t) ; p\right], \\
& =N_{0} \int_{0}^{\infty} e^{-p t} \sum_{k=0}^{\infty} \frac{(-c)^{k}}{k ! \Gamma(l+k+(b+1) / 2)} \\
& \times\left(\frac{d^{v} t^{\nu}}{2}\right)^{2 k+l}-d^{v} p^{-v} N(p), \\
& N(p)\left[1+d^{v} p^{-v}\right] \\
& =N_{0} \sum_{k=0}^{\infty} \frac{(-c)^{k}\left(d^{v} / 2\right)^{2 k+l}}{k ! \Gamma(l+k+(b+1) / 2)} \int_{0}^{\infty} e^{-p t} t^{2 k v+\nu l+1-1} d t \\
& =N_{0} \sum_{k=0}^{\infty} \frac{(-c)^{k}\left(d^{v} / 2\right)^{2 k+l}}{k ! \Gamma(l+k+(b+1) / 2)} \frac{\Gamma(2 k v+v l+1)}{p^{2 k v+v l+1}}, \\
& =N_{0} \sum_{k=0}^{\infty} \frac{(-c)^{k}\left(d^{v} / 2\right)^{2 k+l} \Gamma(2 k v+v l+1)}{k ! \Gamma(l+k+(b+1) / 2)} \\
& \times\left\{p^{-(2 k v+\nu l+1)} \sum_{r=0}^{\infty} \frac{(1)_{r}\left[-(p / d)^{-\nu}\right]^{r}}{(r) !}\right\} .
\end{aligned}
$$

Taking Laplace inverse of (32) and using $L^{-1}\left\{p^{-\nu}\right\}=t^{\nu-1} / \Gamma(\nu)$, $\mathfrak{R}(\nu)>0$, we have

$$
\begin{aligned}
L^{-1}\{N(p)\} \\
=N_{0} \sum_{k=0}^{\infty} \frac{(-c)^{k}\left(d^{v} / 2\right)^{2 k+l} \Gamma(2 k v+v l+1)}{k ! \Gamma(l+k+(b+1) / 2)} \\
\quad \times L^{-1}\left\{\sum_{r=0}^{\infty}(-1)^{r} d^{v r} p^{-(2 k v+v l+v r+1)}\right\},
\end{aligned}
$$

$N(t)$

$$
\begin{aligned}
= & N_{0} \sum_{k=0}^{\infty} \frac{(-c)^{k}\left(d^{\nu} / 2\right)^{2 k+l} \Gamma(2 k v+\nu l+1)}{k ! \Gamma(l+k+(b+1) / 2)} \\
& \times\left\{\sum_{r=0}^{\infty}(-1)^{r} d^{\nu r} \frac{t^{\nu(2 k+l+r)}}{\Gamma(\nu r+2 k \nu+\nu l+1)}\right\}
\end{aligned}
$$




$$
\begin{aligned}
= & N_{0} \sum_{k=0}^{\infty} \frac{(-c)^{k}\left(d^{\nu} / 2\right)^{2 k+l} \Gamma(2 k v+v l+1)}{k ! \Gamma(l+k+(b+1) / 2)} t^{\nu(2 k+l)} \\
& \times\left\{\sum_{r=0}^{\infty}(-1)^{r} d^{\nu r} \frac{t^{\nu r}}{\Gamma(\nu r+2 k v+\nu l+1)}\right\} \\
= & N_{0} \sum_{k=0}^{\infty} \frac{(-c)^{k} \Gamma(2 k v+\nu l+1)}{k ! \Gamma(l+k+(b+1) / 2)}\left(\frac{d^{\nu} t^{\nu}}{2}\right)^{2 k+l} \\
& \times\left\{\sum_{r=0}^{\infty}(-1)^{r} d^{\nu r} \frac{t^{\nu r}}{\Gamma(\nu(r+2 k+l)+1)}\right\},
\end{aligned}
$$

$N(t)$

$$
\begin{aligned}
= & N_{0} \sum_{k=0}^{\infty} \frac{(-c)^{k} \Gamma(2 k v+v l+1)}{k ! \Gamma(l+k+(b+1) / 2)}\left(\frac{d^{v} t^{\nu}}{2}\right)^{2 k+l} \\
& \times E_{\nu,(2 k+l) v+1}\left(-d^{v} t^{\nu}\right) .
\end{aligned}
$$

This completes the proof of Theorem 5.

If we set $b=c=1$ in Theorem 5 , then generalized Bessel function $\omega_{l, b, c}(z)$ reduces to Bessel function of the first kind $J_{l}(z)$, and we arrive at the special case of (28).

Corollary 6. If $d>0, v>0, l, t \in \mathbb{C}$, and $\mathfrak{R}(l)>-1$, then for the solution of the equation

$$
N(t)-N_{0} J_{l}\left(d^{\nu} t^{\nu}\right)=-d^{\nu}{ }_{0} D_{t}^{-\nu} N(t),
$$

the following result holds:

$$
\begin{aligned}
N(t)= & N_{0} \sum_{k=0}^{\infty} \frac{(-1)^{k} \Gamma(2 k v+v l+1)}{k ! \Gamma(l+k+1)}\left(\frac{d^{\nu} t^{\nu}}{2}\right)^{2 k+l} \\
& \times E_{\nu,(2 k+l) v+1}\left(-d^{\nu} t^{\nu}\right) .
\end{aligned}
$$

On taking $b=1$ and $c=-1$ in (28), then generalized Bessel function $\omega_{l, b, c}(z)$ reduces to Bessel function of the first kind $J_{l}(z)$, and we get the following result.

Corollary 7. If $d>0, v>0, l, t \in \mathbb{C}$, and $\mathfrak{R}(l)>-1$, then for the solution of the equation

$$
N(t)-N_{0} I_{l}\left(d^{v} t^{v}\right)=-d^{v}{ }_{0} D_{t}^{-v} N(t),
$$

the following result holds:

$$
\begin{aligned}
N(t)= & N_{0} \sum_{k=0}^{\infty} \frac{\Gamma(2 k v+v l+1)}{k ! \Gamma(l+k+1)}\left(\frac{d^{v} t^{\nu}}{2}\right)^{2 k+l} \\
& \times E_{\nu,(2 k+l) v+1}\left(-d^{\nu} t^{\nu}\right) .
\end{aligned}
$$

Further, if we put $b=2$ and $c=1$ in (28), then we arrive at the following interesting result.

Corollary 8. If $d>0, v>0, l, t \in \mathbb{C}$, and $\Re(l)>-1$, then for the solution of the equation

$$
N(t)-N_{0} j_{l}\left(d^{v} t^{v}\right)=-d^{v}{ }_{0} D_{t}^{-v} N(t)
$$

the following result holds:

$$
\begin{aligned}
N(t)= & N_{0} \sqrt{\frac{\pi}{2}} \sum_{k=0}^{\infty} \frac{(-1)^{k} \Gamma(2 k v+v l+1)}{k ! \Gamma(l+k+3 / 2)}\left(\frac{d^{v} t^{\nu}}{2}\right)^{2 k+l} \\
& \times E_{v,(2 k+l) v+1}\left(-d^{v} t^{\nu}\right),
\end{aligned}
$$

where $j_{l}(z)$ is the spherical Bessel function of the first kind.

Theorem 9. If $a>0, d>0, v>0, c, b, l, t \in \mathbb{C}, a \neq d$, and $\mathfrak{R}(l)>-1$, then for the solution of the equation

$$
N(t)-N_{0} \omega_{l, b, c}\left(d^{v} t^{v}\right)=-a^{v}{ }_{0} D_{t}^{-v} N(t),
$$

there holds the formula:

$$
\begin{aligned}
N(t)= & N_{0} \sum_{k=0}^{\infty} \frac{(-c)^{k} \Gamma(2 k v+v l+1)}{k ! \Gamma(l+k+(b+1) / 2)}\left(\frac{d^{v} t^{\nu}}{2}\right)^{2 k+l} \\
& \times E_{v,(2 k+l) v+1}\left(-a^{\nu} t^{\nu}\right) .
\end{aligned}
$$

Proof. Applying the Laplace transform to both sides of (40), we get

$$
\begin{aligned}
& L[N(t) ; p] \\
& =N_{0} L\left[\omega_{l, b, c}\left(d^{v} t^{\nu}\right) ; p\right]-a^{\nu} L\left[{ }_{0} D_{t}^{-v} N(t) ; p\right], \\
& N(p)\left[1+a^{v} p^{-v}\right] \\
& =N_{0} \sum_{k=0}^{\infty} \frac{(-c)^{k}\left(d^{v} / 2\right)^{2 k+l}}{k ! \Gamma(l+k+(b+1) / 2)} \frac{\Gamma(2 k v+v l+1)}{p^{2 k v+v l+1}}, \\
& N(p) \quad \\
& =N_{0} \sum_{k=0}^{\infty} \frac{(-c)^{k}\left(d^{v} / 2\right)^{2 k+l} \Gamma(2 k v+v l+1)}{k ! \Gamma(l+k+(b+1) / 2)} \\
& \quad \times\left\{p^{-(2 k v+v l+1)} \sum_{r=0}^{\infty} \frac{(1)_{r}(-1)^{r} p^{-v r} a^{v r}}{(r) !}\right\} .
\end{aligned}
$$


Taking Laplace inverse of (42), we arrive at

$$
\begin{aligned}
L^{-1}\{N(p)\} \\
=N_{0} \sum_{k=0}^{\infty} \frac{(-c)^{k}\left(d^{v} / 2\right)^{2 k+l} \Gamma(2 k v+v l+1)}{k ! \Gamma(l+k+(b+1) / 2)} \\
\quad \times L^{-1}\left\{\sum_{r=0}^{\infty}(-1)^{r} a^{v r} p^{-(2 k v+v l+v r+1)}\right\},
\end{aligned}
$$

$N(t)$

$$
\begin{aligned}
= & N_{0} \sum_{k=0}^{\infty} \frac{(-c)^{k} \Gamma(2 k \nu+\nu l+1)}{k ! \Gamma(l+k+(b+1) / 2)}\left(\frac{d^{\nu} t^{\nu}}{2}\right)^{2 k+l} \\
& \times\left\{\sum_{r=0}^{\infty}(-1)^{r} a^{\nu r} \frac{t^{\nu r}}{\Gamma(\nu(r+2 k+l)+1)}\right\},
\end{aligned}
$$

$N(t)$

$$
\begin{aligned}
= & N_{0} \sum_{k=0}^{\infty} \frac{(-c)^{k} \Gamma(2 k \nu+\nu l+1)}{k ! \Gamma(l+k+(b+1) / 2)}\left(\frac{d^{v} t^{\nu}}{2}\right)^{2 k+l} \\
& \times E_{\nu,(2 k+l) v+1}\left(-a^{\nu} t^{\nu}\right) .
\end{aligned}
$$

This completes the proof of Theorem 9.

Remark 10. The special cases for Theorem 9 can be developed on similar lines to that of Corollaries 6-8, but we do not state here due to lack of space.

\section{Conclusion}

In this paper we have studied a new fractional generalization of the standard kinetic equation and derived solutions for it. It is not difficult to obtain several further analogous fractional kinetic equations and their solutions as those exhibited here by main results. Moreover, by the use of close relationships of the generalized Bessel function of the first kind $\omega_{p}(z)$ with many special functions, we can easily construct various known and new fractional kinetic equations.

\section{Conflict of Interests}

The authors declare that there is no conflict of interests regarding the publication of this paper.

\section{Acknowledgment}

The authors are thankful to the referee for the very careful reading and the valuable suggestions.

\section{References}

[1] Á. Baricz, Generalized Bessel Functions of the First Kind, vol. 1994 of Lecture Notes in Mathematics, Springer, Berlin, Germany, 2010.

[2] Á. Baricz, "Geometric properties of generalized Bessel functions," Publicationes Mathematicae Debrecen, vol. 73, no. 1-2, pp. 155-178, 2008.

[3] J. Choi, P. Agarwal, S. Mathur, and S. D. Purohit, "Certain new integral formulas involving the generalized Bessel functions," Bulletin of the Korean Mathematical Society, vol. 51, no. 4, Article ID 9951003, 2014.

[4] P. Malik, S. R. Mondal, and A. Swaminathan, "Fractional integration of generalized bessel function of the first kind," in Proceedings of the ASME International Design Engineering Technical Conferences and Computers and Information in Engineering Conference (IDETC/CIE '11), pp. 409-418, Washington, DC, USA, August 2011.

[5] S. R. Mondal and K. S. Nisar, "Marichev-Saigo-Maeda fractional integration operators involving generalized Bessel functions," Mathematical Problems in Engineering, vol. 2014, Article ID 274093, 11 pages, 2014.

[6] D. Baleanu, P. Agarwal, and S. D. Purohit, "Certain fractional integral formulas involving the product of generalized Bessel functions," The Scientific World Journal, vol. 2013, Article ID 567132, 9 pages, 2013.

[7] G. N. Watson, A Treatise on the Theory of Bessel Functions, Cambridge University Press; The Macmillan, Cambridge, UK, 1944.

[8] J. K. Prajapat, "Certain geometric properties of normalized Bessel functions," Applied Mathematics Letters, vol. 24, no. 12, pp. 2133-2139, 2011.

[9] L. Galué, "A generalized Bessel function," Integral Transforms and Special Functions, vol. 14, no. 5, pp. 395-401, 2003.

[10] E. Deniz, H. Orhan, and H. M. Srivastava, "Some sufficient conditions for univalence of certain families of integral operators involving generalized Bessel functions," Taiwanese Journal of Mathematics, vol. 15, no. 2, pp. 883-917, 2011.

[11] G. M. Zaslavsky, "Fractional kinetic equation for Hamiltonian chaos," Physica D, vol. 76, no. 1-3, pp. 110-122, 1994.

[12] A. I. Saichev and G. M. Zaslavsky, "Fractional kinetic equations: solutions and applications," Chaos, vol. 7, no. 4, pp. 753-764, 1997.

[13] H. J. Haubold and A. M. Mathai, "The fractional kinetic equation and thermonuclear functions," Astrophysics and Space Science, vol. 273, no. 1-4, pp. 53-63, 2000.

[14] R. K. Saxena, A. M. Mathai, and H. J. Haubold, "On fractional kinetic equations," Astrophysics and Space Science, vol. 282, no. 1, pp. 281-287, 2002.

[15] R. K. Saxena, A. M. Mathai, and H. J. Haubold, "On generalized fractional kinetic equations," Physica A, vol. 344, no. 3-4, pp. 657-664, 2004.

[16] R. K. Saxena, A. M. Mathai, and H. J. Haubold, "Solution of generalized fractional reaction-diffusion equations," Astrophysics and Space Science, vol. 305, no. 3, pp. 305-313, 2006.

[17] R. K. Saxena and S. L. Kalla, "On the solutions of certain fractional kinetic equations," Applied Mathematics and Computation, vol. 199, no. 2, pp. 504-511, 2008.

[18] V. B. L. Chaurasia and S. C. Pandey, "On the new computable solution of the generalized fractional kinetic equations involving the generalized function for the fractional calculus and 
related functions," Astrophysics and Space Science, vol. 317, no. 3-4, pp. 213-219, 2008.

[19] V. G. Gupta, B. Sharma, and F. B. M. Belgacem, "On the solutions of generalized fractional kinetic equations," Applied Mathematical Sciences, vol. 5, no. 19, pp. 899-910, 2011.

[20] A. Chouhan and S. Sarswat, "On solution of generalized Kinetic equation of fractional order," International journal of Mathematical Sciences and Applications, vol. 2, no. 2, pp. 813-818, 2012.

[21] A. Chouhan, S. D. Purohit, and S. Saraswat, "An alternative method for solving generalized differential equations of fractional order," Kragujevac Journal of Mathematics, vol. 37, no. 2, pp. 299-306, 2013.

[22] A. Gupta and C. L. Parihar, "On solutions of generalized kinetic equations of fractional order," Boletim da Sociedade Paranaense de Matemática, vol. 32, no. 1, pp. 181-189, 2014.

[23] R. K. Saxena and D. Kumar, "Solution of fractional kinetic equation associated with aleph function," Submitted.

[24] M. R. Spiegel, Theory and Problems of Laplace Transforms, Schaums Outline Series, McGraw-Hill, New York, NY, USA, 1965.

[25] G. Mittag-Leffler, "Sur la représentation analytique d'une branche uniforme d'une fonction monogène," Acta Mathematica, vol. 29, no. 1, pp. 101-181, 1905.

[26] A. Erdélyi, W. Magnus, F. Oberhettinger, and F. G. Tricomi, Tables of Integral Transforms, vol. 1, McGraw-Hill, New York, NY, USA, 1954.

[27] H. M. Srivastava and R. K. Saxena, "Operators of fractional integration and their applications," Applied Mathematics and Computation, vol. 118, no. 1, pp. 1-52, 2001. 


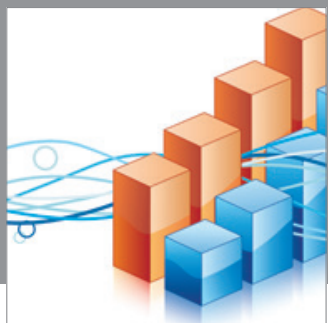

Advances in

Operations Research

mansans

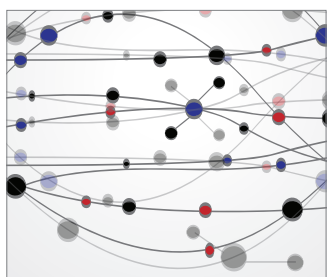

The Scientific World Journal
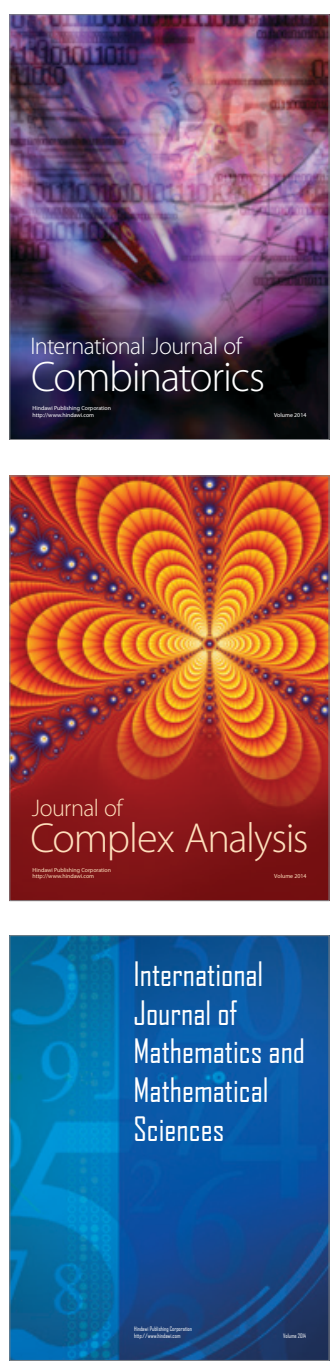
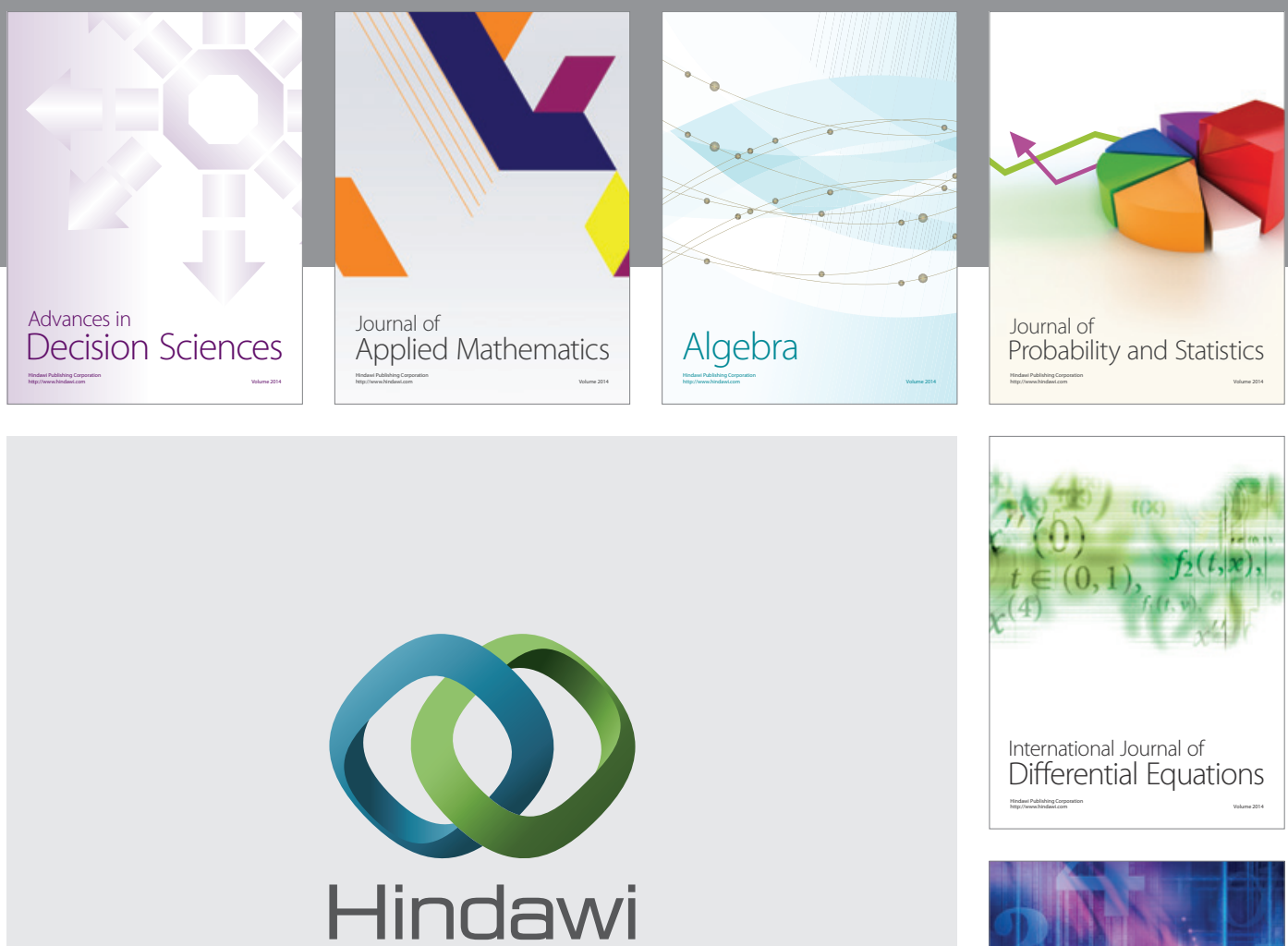

Submit your manuscripts at http://www.hindawi.com
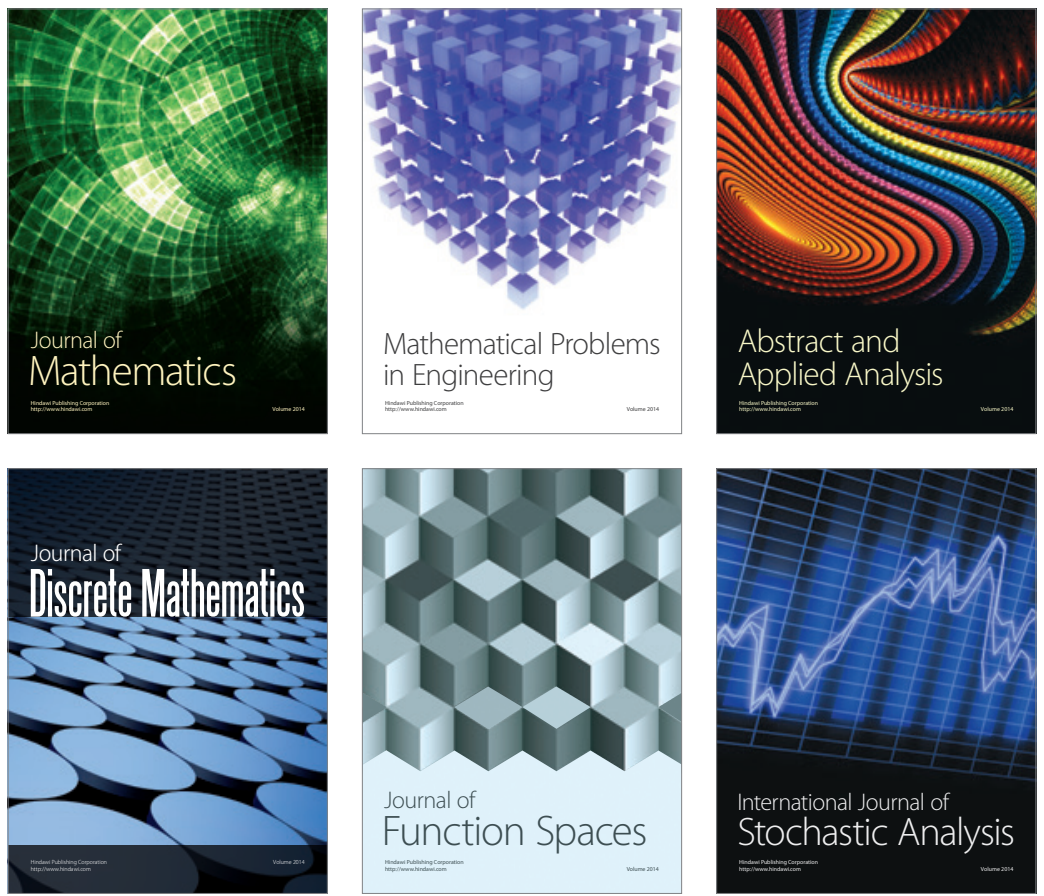

Journal of

Function Spaces

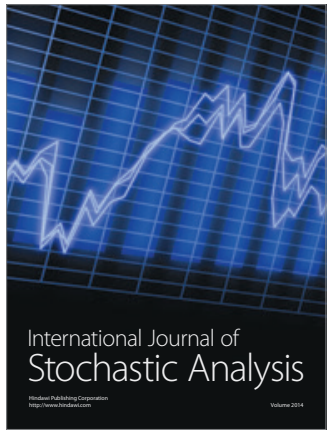

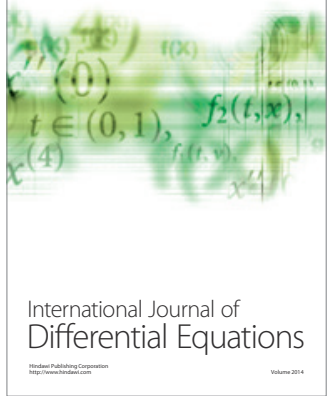
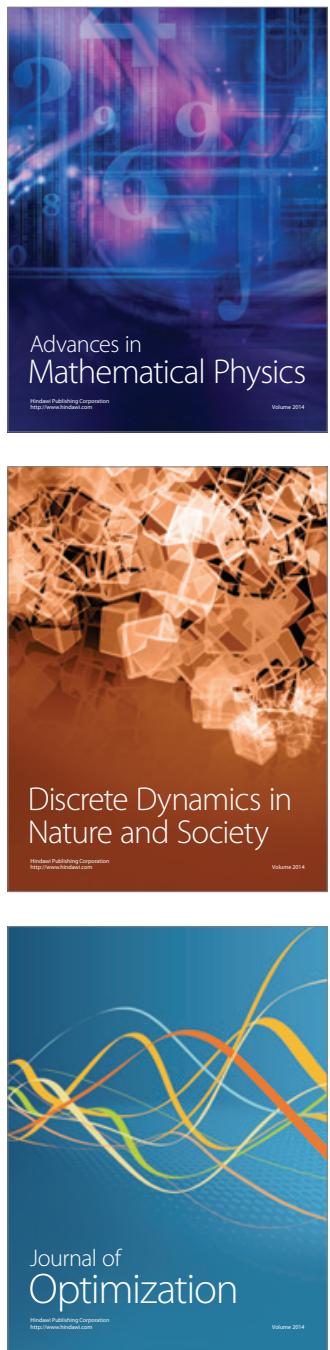